\title{
The Role of Mitochondrial NADPH-Dependent Isocitrate Dehydrogenase in Cancer Cells
}

\author{
Katarína Smolková and Petr Ježek \\ Department of Membrane Transport Biophysics (No. 75), Institute of Physiology v.v.i., Academy of Sciences of the Czech Republic, \\ Vídeňská 1083, CZ-14220 Prague, Czech Republic \\ Correspondence should be addressed to Petr Ježek, jezek@biomed.cas.cz
}

Received 16 January 2012; Accepted 19 March 2012

Academic Editor: Juan P. Bolaños

Copyright ( $\odot 2012$ K. Smolková and P. Ježek. This is an open access article distributed under the Creative Commons Attribution License, which permits unrestricted use, distribution, and reproduction in any medium, provided the original work is properly cited.

Isocitrate dehydrogenase 2 (IDH2) is located in the mitochondrial matrix. IDH2 acts in the forward Krebs cycle as an $\mathrm{NADP}^{+}$-consuming enzyme, providing NADPH for maintenance of the reduced glutathione and peroxiredoxin systems and for self-maintenance by reactivation of cystine-inactivated IDH2 by glutaredoxin 2 . In highly respiring cells, the resulting $\mathrm{NAD}^{+}$accumulation then induces sirtuin-3-mediated activating IDH2 deacetylation, thus increasing its protective function. Reductive carboxylation of 2-oxoglutarate by IDH2 (in the reverse Krebs cycle direction), which consumes NADPH, may follow glutaminolysis of glutamine to 2-oxoglutarate in cancer cells. When the reverse aconitase reaction and citrate efflux are added, this overall "anoxic" glutaminolysis mode may help highly malignant tumors survive aglycemia during hypoxia. Intermittent glycolysis would hypothetically be required to provide ATP. When oxidative phosphorylation is dormant, this mode causes substantial oxidative stress. Arg172 mutants of human IDH2-frequently found with similar mutants of cytosolic IDH1 in grade 2 and 3 gliomas, secondary glioblastomas, and acute myeloid leukemia-catalyze reductive carboxylation of 2-oxoglutarate and reduction to D-2-hydroxyglutarate, which strengthens the neoplastic phenotype by competitive inhibition of histone demethylation and 5-methylcytosine hydroxylation, leading to genome-wide histone and DNA methylation alternations. D-2-hydroxyglutarate also interferes with proline hydroxylation and thus may stabilize hypoxia-induced factor $\alpha$.

\section{Oxidative Phosphorylation and Glutaminolysis in Cancer Cells}

1.1. Strategies for Survival of Malignant Tumors. During malignant transformation, cells undergo stages of gene expression reprogramming and mutagenesis that alter their metabolic phenotype(s) [1-5]. Initial stimuli (not all known) dysregulate information signaling and activate oncogenes and/ or cancer stem cells, resulting in a partial glycolytic "Warburg" phenotype [1-5] in which pyruvate is diverted, at least to a certain extent, from oxidative phosphorylation (OXPHOS). High proliferation and impaired angiogenesis subsequently cause hypoxia in certain regions within a growing tumor, and then hypoxia-mediated metabolic reprogramming (such as that promoted by hypoxia-induced factor, HIF [6-8]) further intensifies the glycolytic phenotype and may nearly completely divert pyruvate from pyruvate dehydrogenase (PDH), that is, from OXPHOS. The sustained high rate of cell proliferation, however, results in aglycemia, initiating the revival of OXPHOS in conjunction with the promotion of glutaminolysis $[1,2,9,10]$. The overall glutaminolysis provides cytosolic pyruvate/lactate and also yields NADPH via citrate export from mitochondria and subsequent ATP-citrate lyase and malic enzyme reactions. This compensates for the reduced net energy production by the glycolytic pathway and pentose phosphate pathway (PPP). Pyruvate imported into mitochondria is the precursor of not only acetyl-CoA but also citrate, which is required for fatty acid synthesis and hence for phospholipid synthesis, so it is essential for cell growth [1-5]. The final established phenotype is exemplified by human glioblastoma cells, which, despite their low respiration, maintain a constant 
pyruvate flux through PDH and hence partial OXPHOS [9]. Oxaloacetate, however, may also be provided by the pyruvate carboxylase reaction [11].

1.2. Glutaminolysis at Rejuvenated OXPHOS. For the purpose of this paper, we shall use the term "glutaminolysis" in a more general way than just the transformation of glutamine to 2-oxoglutarate (2OG). We categorize glutaminolysis according the fate of $2 \mathrm{OG}$ after its initial formation from glutamine [1]. If 2OG resulting from glutamine acts in the forward Krebs cycle (despite possible ongoing citrate extrusion and truncation of the cycle so that aconitase and "classic" $\mathrm{NAD}^{+}$-dependent isocitrate dehydrogenase (IDH3) reactions are not required), we define the system of metabolic reactions involved as "OXPHOS glutaminolysis." This term points out to its essential dependence on succinate dehydrogenase (Complex II) and hence on respiration and OXPHOS. In contrast, when the reductive carboxylation of $2 \mathrm{OG}$ by isocitrate dehydrogenase 2 (IDH2) (in the counter Krebs cycle direction) consuming NADPH follows glutaminolysis of glutamine to 2OG and when the reverse aconitase reaction and citrate efflux are added, we define that system as "reductive carboxylation glutaminolysis" (RCG), also referred to as "anoxic glutaminolysis." The latter term denotes the absolute independence of oxygen (respiration).

In general, glutaminolysis is an anaplerotic pathway of the Krebs cycle. Although it acts frequently in broad cancer types, glutaminolysis is not universal for all cancers [3-5]. In cancer cells employing OXPHOS glutaminolysis, glutamine can fully compensate for the lack of glucose in terms of energy generation and syntheses of precursors for anabolic pathways [3-5]. Thus, to survive under conditions of limited glucose, highly glycolytic cancer cells may adapt to glutaminolysis, which in its OXPHOS mode restores OXPHOS and may restore also at least partial PDH function $[1,3,12-$ 14]. In normal cells, mitochondrial glutaminase catabolizes glutamine to produce ammonia and glutamate, which is further transaminated by glutamate dehydrogenase into $2 \mathrm{OG}$ to feed the Krebs cycle [15]. In malignant tumors, negative allosteric effectors, such as GTP, inhibit glutamate dehydrogenase, resulting in a move toward glutaminolysis, where glutamate and pyruvate are reactants in a transamination reaction that produces, for example, alanine and 2OG by alanine aminotransferase (transaminase) [15]. In cancer cells, 2OG usually feeds the forward-running Krebs cycle truncated after citrate synthase during citrate extrusion, so that aconitase and "classic" $\mathrm{NAD}^{+}$-dependent IDH3 reactions are not required $[1,5]$. This OXPHOS glutaminolytic mode is strictly dependent on oxaloacetate and acetyl-CoA, that is, on the citrate synthase reaction; hence, it proceeds only in cells in which oxaloacetate is provided by malate dehydrogenase fed by the Krebs cycle as well as by malate import from the cytosol, where malate originates from ATPcitrate lyase reaction. Likewise incomplete inhibition of $\mathrm{PDH}$ restores acetyl-CoA in mitochondria (the pyruvate pool is split between the PDH and transaminase reactions). An alternative oxaloacetate source is provided by pyruvate carboxylase reaction [11].
Also, the mitochondrial malic enzyme may contribute to this pool by producing pyruvate from malate [16]. Citrate is extruded from mitochondria and converted to oxaloacetate and acetyl-CoA by ATP citrate lyase [17]. Acetyl-CoA is then used to produce fatty acids by fatty acid synthase and cholesterol for general lipid synthesis, which is essential for cancer cell proliferation $[18,19]$. In glioblastoma, if there is excess NADH in the cytoplasm (produced by aerobic glycolysis), the cytosolic oxaloacetate is converted first to malate by malate dehydrogenase and then to pyruvate by the cytosolic malic enzyme, thus may also contribute to lactate production $[9,20]$. The cytosolic malic enzyme also produces NADPH as another factor required for lipid biosynthesis. Moreover, alanine that is released by transaminase is used for cytosolic amino acid transformations and protein synthesis $[1,4,5]$.

1.3. Glutaminolysis Independent of OXPHOS. Hypothetically, malignant tumors may survive on intermittent OXPHOSindependent RCG (also termed "anoxic" glutaminolysis) in parallel with intermittent glycolytic periods $[1,2]$. RCG utilizes reductive carboxylation of $2 \mathrm{OG}$ by the reverse reaction of mitochondrial IDH2 at the expense of NADPH, followed by the reverse aconitase reaction and citrate efflux from the matrix $[1-3,21-23]$. NADPH is provided by the malic enzyme converting malate to pyruvate and might also be provided by the mitochondrial transhydrogenase [24]. The OXPHOS independence of this mode means that it may proceed at any level of hypoxia and even at anoxia, thus increasing malignancy $[1,3]$. However, it does not produce ATP so parallel glycolytic periods are required [1]. The reductive carboxylation involves IDH2, which converts $2 \mathrm{OG}$ to isocitrate, from which the reverse aconitase reaction produces citrate, which is again exported from the mitochondrial matrix to the cytosol for fatty acid and lipid synthesis. Note that acetyl-CoA, and hence the PDH reaction, is not required in this mode.

\section{Isocitrate Dehydrogenase Enzyme Isoforms}

2.1. Overview of IDH Isoforms. All eukaryot genomes contain three IDH genes. IDH3 encodes a mitochondrial matrix $\mathrm{NAD}^{+}$-dependent octameric IDH3 ( $4 \alpha 2 \beta 2 \gamma$ subunits [25]) that acts in the Krebs cycle. IDH3 is allosterically positively regulated by $\mathrm{Ca}^{2+}$, ADP, and citrate and negatively regulated by ATP, NADH, and NADPH. The two other IDH genes, $I D H 1$ and $I D H 2$, encode cytosolic and mitochondrial matrix $\mathrm{NADP}^{+}$-dependent (or NADPH-dependent) IDH1 and IDH2, respectively, which are structurally and genetically unrelated to IDH3 [26] (Table 1). IDH3 irreversibly decarboxylates isocitrate to yield 2OG while reducing $\mathrm{NAD}^{+}$ to $\mathrm{NADH}$, whereas IDH1 and IDH2 catalyze reversible reactions, either decarboxylating isocitrate to 2OG while reducing $\mathrm{NADP}^{+}$to NADPH or acting in the reductive carboxylation reaction to convert $2 \mathrm{OG}$ to isocitrate while oxidizing NADPH to $\mathrm{NADP}^{+}$.

Heterozygous mutations in $I D H 2$ at $\operatorname{Arg} 172$ and at the analogous residue Arg132 in IDH1 are frequently found in grade 2 and 3 gliomas, secondary glioblastomas, and 
TABLE 1: Kinetics of IDH isoforms as compared to homodimeric mutant enzymes with clinically relevant mutations. Unless specified "reductive", forward reactions were measured. "n.d.”: not determined.

\begin{tabular}{|c|c|c|c|c|c|c|c|c|}
\hline Enzyme & Organism/tissue & Mutation & conditions & $\begin{array}{c}\mathrm{K}_{m} \\
\text { isocitrate } \\
(\mu \mathrm{M})\end{array}$ & $\begin{array}{c}\mathrm{K}_{l}, 2 \text {-oxo- } \\
\text { glutarate } \\
(\mu \mathrm{M})\end{array}$ & $\begin{array}{c}\mathrm{K}_{m} \mathrm{NADP}^{+} \\
(\mu \mathrm{M})\end{array}$ & $\begin{array}{c}V_{\max } \\
\left(\mu \mathrm{mol} \cdot \min ^{-1} \cdot \mathrm{mg}^{-1}\right)\end{array}$ & Reference \\
\hline \multirow{6}{*}{ IDH1 } & Human & wt & \multirow{6}{*}{ Reductive } & 65 & 1900 & 49 & $44000 \mathrm{~s}^{-1}$ & {$[96]$} \\
\hline & Human & $\mathrm{R} 132 \mathrm{H}$ & & 370 & 24 & 84 & $38 \mathrm{~s}^{-1}$ & {$[96]$} \\
\hline & Human & $\mathrm{R} 132 \mathrm{H}$ & & 2OG: 965 & n.d & $\begin{array}{l}\text { NADPH: } \\
0.44\end{array}$ & $1000 \mathrm{~s}^{-1}$ & {$[96]$} \\
\hline & Human & wt & & 6.4 & n.d. & n.d. & 14 & {$[101]$} \\
\hline & Human & $\mathrm{R} 132 \mathrm{H}$ & & 1280 & n.d. & n.d. & 0.8 & {$[101]$} \\
\hline & Rat liver & wt & & 120 & n.d. & 150 & 70 & [102] \\
\hline \multirow{6}{*}{ IDH2 } & Porcine & wt & & 8.4 & n.d. & 5.6 & 43 & {$[103]$} \\
\hline & Porcine & wt & & 6 & n.d. & 5 & 40 & {$[51]$} \\
\hline & Porcine & R133Q & & 990 & n.d. & 11 & 21 & {$[51]$} \\
\hline & Rat liver & wt & & 70 & n.d. & 60 & 66 & {$[102]$} \\
\hline & Rat heart & wt & & 45 & 80 & 46 & 16 & {$[104]$} \\
\hline & Rat heart & wt & Ischemic & 17 & 250 & 46 & 38 & {$[104]$} \\
\hline \multirow{2}{*}{ IDH3 } & Human & wt & No ADP & 2000 & n.d. & $\mathrm{NAD}^{+}: 60$ & 26 & {$[105]$} \\
\hline & Human & wt & $+1 \mathrm{mM}$ ADP & 50 & n.d. & n.d. & n.d. & [105] \\
\hline
\end{tabular}

acute myeloid leukemia (AML [27]), but they occur less frequently in primary glioblastomas and other cancers [2837]. No homozygous deletions of IDH1 and IDH 2 have been found, as has been observed for classic tumor suppressors. Nevertheless, mutated IDH1 and IDH2 exhibit a neomorphic enzyme activity, reducing $2 \mathrm{OG}$ to D-2-hydroxyglutarate while converting NADPH to $\operatorname{NADP}^{+}[23,29,36,38-40]$. Interestingly, the D-2-hydroxyglutarate thus formed further promotes neoplasia by competitive inhibition of histone demethylation and 5-methyl-cytosine hydroxylation, leading to genome-wide alternations in the methylation of histones and DNA [40]. It has also been reported that glioblastoma SF188 cells produce D-2-hydroxyglutarate, in spite of lacking the above-described mutations [41].

Moreover, IDH2, like $\sim 20 \%$ of other mitochondrial enzymes [42, 43], is acetylated at lysines, which inactivates the enzymatic activity. In turn, deacetylation of IDH2 by the mitochondrial matrix deacetylase sirtuin 3 (SIRT3) activates the enzyme to produce more NADPH [44]. In nonmalignant cells, the cytosolic IDH1 is involved in lipid metabolism and glucose sensing. IDH2 was traditionally considered to be involved in the regulation of OXPHOS and redox homeostasis [45] (see Section 4), and its involvement in reductive carboxylation has been recognized only recently (Sections 2.4 and 3 ).

2.2. Specific Enzymatic Properties of IDH2. $\mathrm{NADP}^{+}$-dependent oxidative decarboxylation of isocitrate to 2OG, as the major function of IDH2 in nonmalignant cells, contributes substantially to the control of mitochondrial redox balance and the prevention of oxidative damage $[45,46]$. Because the IDH2 reaction is reversible, it may act in a "reverse" Krebs cycle mode in the reductive carboxylation reaction (see Section 2.4). IDH2 contains an N-terminal mitochondrial addressing sequence and hence is imported to the mitochondrial matrix [45], although localization to nuclei has also been reported [47]. The IDH2 locus is adjacent to the gene for the $\alpha$ subunit of IDH3 [48]. IDH2 expression in heart, skeletal muscle, and lymphocytes is quite substantial; lower levels are found in liver, kidney, and lung [45, 47]. IDH2 has also been found in cultured rat neurons, astrocytes, oligodendrocytes, and microglia [49]. Unlike IDH1, the 94$\mathrm{kDa}$ IDH2 (EC 1.1.1.42) is a homodimeric enzyme of two 413-amino acid subunits, each $47 \mathrm{kDa}[50,51]$ (Figure 1). IDH2 function requires a divalent metal ion, and bound $\mathrm{Mn}^{2+}$ yields the maximum activity [52]. The structure of the $\mathrm{Mn}^{2+}$-isocitrate binding site was mapped from the solved crystal structure of porcine IDH2 [50]. Within the site, Thr78, Ser95, and Asn97 (of the porcine sequence) donate a hydrogen bond to the C3 carboxyl, whereas Asp252 and Asp275 coordinate $\mathrm{Mn}^{2+}$. An additional six Arg residues provide hydrogen bonds with isocitrate oxygens [50]. Hydrogen bonding of Lys 212 with other residues between the two subunits was also noted. The $\mathrm{NADP}^{+}$binding site was originally predicted from the E. coli IDH structures, positioning the 2-hydroxyl-bound phosphate to interact with His315 and Lys374 of porcine IDH2 [50]. Porcine Arg83 enhances $\mathrm{NADP}^{+}$affinity by hydrogen bonding with the 3 - $-\mathrm{OH}$ of the nicotinamide ribose, and Asn 328 provides a hydrogen bond to the N1 of adenine [53]. For efficient coenzyme site function, a hydroxyl group must be present at position 373 (Thr373 of the porcine sequence), whereas Asp375 and Lys 260 contribute to coenzyme affinity and catalysis $[54,55]$.

Within the numbering of the human IDH2 sequence, mutations in Arg172 (an analog of frequently mutated Arg132 of cytosolic IDH1 [56]) are detected in gliomas $[23,36,37]$, and mutations in Arg172 and Arg140 (which 


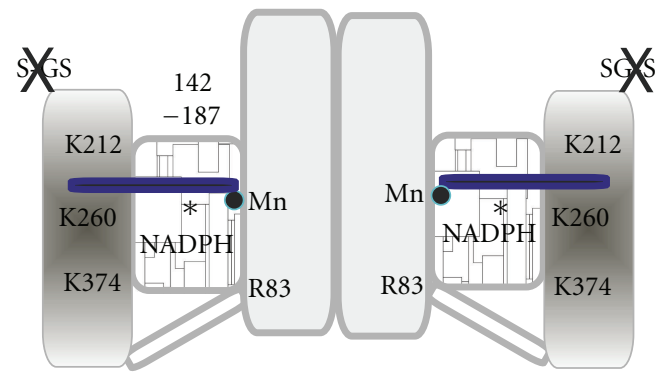

Figure 1: Main domains of the mitochondrial isocitrate dehydrogenase, IDH2.The homodimeric IDH2 of two 413-amino acid subunits; each $47 \mathrm{kDa}$ subunit is schematically illustrated according the solved crystal structure of porcine IDH2 [50]. Residues 142-187 make up two stacked four-stranded antiparallel $\beta$-sheets (middle white marble-shaded domain). $\mathrm{Mn}^{2+}$ and isocitrate in the active site are depicted as a blue circle and rod, respectively. Six Arg residues, which provide hydrogen bonds with isocitrate oxygens [50], are not depicted. Arg 83 (R83) interacts by hydrogen bonding with the 3r$\mathrm{OH}$ of the nicotinamide ribose and thus enhances $\mathrm{NADP}^{+}$affinity [53]. An Arg residue corresponding to human Arg172, which is often mutated in gliomas and AML, is indicated by an asterisk $(*)$. For activity, the glutathionylation of Cys269 [46] (GS-S) must be removed $(\mathrm{X})$ by reactivation aided by glutaredoxin-2 in the presence of reduced glutathionine, and the protein must be deacetylated by SIRT3 [44]. We hypothesize that Lys212, Lys374, and Lys260 (in porcine notation) are the prime candidates for acetylation causing IDH2 inactivation.

is adjacent in the active site to $\operatorname{Arg} 172$ [50]) are found in AML [57]. Mutations are apparent after the transition from a normal cell to a clinically evident tumor. Arg172 (as well as Arg132 of IDH1) provides hydrogen bonds to the $\alpha$ and $\beta$ carboxyls of isocitrate and may be important in the transition from an open to closed state of the active site [50]. The porcine Arg residue mutated to Asn in a position analogous to human Arg172 displays a twofold reduction in specific activity and a $\mathrm{K}_{m}$ for isocitrate that is two orders of magnitude higher [51] (Table 1). Likewise, in lysates of cells overexpressing IDH2, activity is reduced when Arg172 is substituted with Gly, Lys, or Met [37]. Nevertheless, the existence of exclusively heterozygous IDH2 (and IDH1) mutations in gliomas and AML and the small possibility of dominant-negative mutations (minor mutant fractions existing could not exert this role [39]) have led to the search for other consequences of IDH1 and IDH2 mutations.

Arg132 mutants of cytosolic IDH1 [27] (Table 1) and Arg172 mutants of mitochondrial IDH2 [23, 36, 38, 39] possess the ability to reduce $2 \mathrm{OG}$ to $\mathrm{D}$-2-hydroxyglutarate while converting NADPH to $\mathrm{NADP}^{+}$. This is because the active closed state of the enzyme exhibits a higher affinity for NADPH. Mutant IDH2 and IDH1 [27] are not supposed to allow the reductive carboxylation reaction of $2 \mathrm{OG}$ to isocitrate. However, consequences of these mutations have appeared more detrimental than expected. Initially, the production of $\mathrm{D}$-2-hydroxyglutarate in gliomas, secondary glioblastomas, and AML by mutant IDH2 results in a decrease of $2 \mathrm{OG}$ and hence depletion of succinate, fumarate, and malate from the rest of the Krebs cycle $[23,36,39]$.
Interestingly, IDH2 mutations also lead to increases in amino acid levels [35] as would be expected for ongoing glutaminolysis. However, the most profound consequences stem from the interference with epigenomics. Thus, the formed D-2-hydroxyglutarate strengthens the neoplastic phenotype by competitive inhibition of histone demethylation and 5-methyl-cytosine hydroxylation, leading to genome-wide alternations in histone and DNA methylation [40]. Moreover, prolyl hydroxylase domain enzymes, which employ $2 \mathrm{OG}$ as a cofactor for marking hypoxia-induced factor- $1 \alpha$ (HIF1 $\alpha$ ) by proline hydroxylation, are inhibited by $\mathrm{D}-2-$ hydroxyglutarate as well as by the lack of 2OG. As a result, HIF1 $\alpha$ is stabilized (if its inhibitor, aspartyl hydroxylase factor inhibiting HIF, is not active) even at normoxia and thus can elicit the otherwise hypoxic reprogramming of gene expression [58].

2.3. Regulation of IDH2 Activity. Glutathionylation of proteins by the reversible glutaredoxin (thioltransferase) reaction serves to protect against irreversible oxidation of cysteines [59]. Such protection has been demonstrated for IDH2 in that oxidized glutathione can inactivate IDH2 by forming a mixed disulfide bond with Cys269 [46]. The inactivated IDH2 is reactivated by glutaredoxin 2 in the presence of reduced glutathione. Also, IDH2 in mouse heart may exist in complex with calcineurin, containing as well aconitase, malate dehydrogenase, and MnSOD [55].

Another important level of regulation of IDH2 activity is provided by mitochondrial SIRT3. Posttranslational modifications of numerous mitochondrial proteins frequently occur via acetylation/deacetylation of lysine residues [42, 43, 60,61 ]. Among seven sirtuin family members, SIRT3, SIRT4, and SIRT5 are enriched in mitochondria, such as exemplified and well described for SIRT3 up to date [42, 43, 62]. It has been reported that incubation of SIRT3 with IDH2 increases the dehydrogenase activity [62]. Recently, caloric restriction was demonstrated to act via IDH2 deacetylation through SIRT3 [44]. Caloric restriction prevents age-related hearing loss by reducing oxidative DNA damage, but such is not the case for mice lacking SIRT3 [44]. SIRT3 directly deacetylates the IDH2 lysines and thus activates IDH2, which in its forward mode could result in increased NADPH levels and thereby maintain reduced glutathione levels in mitochondria. Indeed, overexpression of SIRT3 and/or IDH2 leads to increased NADPH levels and is protective against oxidative stress-induced cell death [44]. Lys212, Lys374, and Lys260 (porcine sequence) may be the prime candidates for acetylation/inactivation. It remains to be shown that SIRT3 can deacetylate these residues, however, and it should be investigated which reaction modes are active and possible before and after such activation, specifically, whether the reverse, NADPH-dependent, reaction and RCG could be activated.

2.4. Reductive Carboxylation Reaction. As predicted in 1994 by Sazanov and Jackson [63] (see also [64]), the reductive carboxylation reaction by native IDH2 converts $2 \mathrm{OG}$ to isocitrate while oxidizing NADPH to $\mathrm{NADP}^{+}$. The Arg172 
and Arg140 mutants of IDH2 [23, 36, 38, 39] and glioblastoma SF188 cells under hypoxia [41] convert 2OG to D2 -hydroxy-glutarate in this "reverse-reaction" mode. This reductive carboxylation would proceed better in vivo when followed by the reverse aconitase reaction and subsequent citrate export from the mitochondrial matrix. Reductive carboxylation was demonstrated for IDH2 in 2002 [65] and was indicated for cancer cells in transformed brown adipocytes [22], pediatric glioma SF188 cells [23, 41], and UOK262 cells (derived from a renal tumor in a patient with hereditary leiomyomatosis, these cells are defective in respiration and devoid of fumarate hydratase activity) [2]. Reductive carboxylation accompanied by citrate efflux has also been found in quiescent fibroblasts and is enhanced in contact-inhibited fibroblasts [66]. IDH2 silencing in SF188 cells results in diminished conversion of glutamine to citrate $[23,41]$. Recently, reductive carboxylation was detected in human osteosarcoma 143B cells in which the mitochondrial DNA encoded a loss-of-function mutation in respiratory chain Complex III (CYTB 143B cells) [2]. Because only lowlevel reductive carboxylation was detected in wild-type $143 \mathrm{~B}$ cells, the authors suggested that the impairment of OXPHOS, such as given by mutant mitochondrial DNA, induces RCG. Silencing of either IDH1 or IDH2 reduces the growth of both wild-type and CYTB 143B cells [2]. Moreover, unlike in wildtype $143 \mathrm{~B}$ cells, de novo fatty acid synthesis from glutamine as a precursor is prevalent in CYTB 143B cells [2]. Reductive carboxylation in fumarate hydratase-devoid UOK262 cells, which are defective in respiration, has also been identified in parallel with OXPHOS glutaminolysis [2]. Interestingly, inhibition of respiration in mouse embryonic fibroblasts via administration of antimycin, rotenone, or metformin induces a switch towards RCG [2]. Thus, these data provide additional support for the authors' hypothesis that RCG is a common cellular response to impaired mitochondrial metabolism [2].

\section{Contribution of IDH2 to Glutaminolysis That Is Independent of OXPHOS}

3.1. Evidence for Reverse Reactions in the Krebs Cycle. The reverse IDH2 reaction was also considered such that IDH2 acts together with the forward reaction of IDH3 in a dissipative isocitrate/2OG cycle [63] (see Section 4.2). The reductive carboxylation reaction and the overall RCG may indeed proceed together with the forward decarboxylation reaction $[2,22]$. The best evidence was obtained by tracking the metabolites of ${ }^{13} \mathrm{C}$-labeled glutamine, such as the appearance of ${ }^{13} \mathrm{C}$-label in citrate $[2,22,23,41]$.

3.2. RCG in Cancer Cells. The first demonstrations of RCG in cancer cells $[22,23,41]$ are consistent with the recent findings that mutant IDH2 in gliomas and AML also produce D-2-hydroxyglutarate from $2 \mathrm{OG}$ by "alternate" reduction. Because these mutants are heterozygous, both RCG and the production of $\mathrm{D}$-2-hydroxyglutarate might occur. The former reaction involves the nonmutant NADPH-dependent IDH2 "reverse" reaction followed by isocitrate conversion to citrate and by citrate export. The mutant IDH2 (but maybe also wild-type IDH2, see [41]) acting in a "reverse" mode also produces $\mathrm{D}$-2-hydroxyglutarate, which cannot be transformed by aconitase; however, it further enhances the malignant phenotype [40]. The importance of this neomorphic IDH2 activity for the cancer phenotype is valid even without consideration of $\mathrm{D}$-2-hydroxyglutarate interference with epigenetics and the HIF pathway, because IDH2 depletes $2 \mathrm{OG}$ from the Krebs cycle.

The consumption of NADPH in the matrix is a consequence of the altered homeostasis of reactive oxygen species (ROS) in cancer cells (see Section 4.2) and is also possible due to NADPH production by the mitochondrial malic enzyme $[1,5]$ and transhydrogenase $[24,63$, 67]. It is not known whether SIRT3-based activation also affects this reverse (NADPH-dependent) IDH2 reaction. Although $\mathrm{NADH}$, rather than $\mathrm{NAD}^{+}$, accumulates in the mitochondrial matrix of highly glycolytic cancer cells in which OXPHOS is dormant (Figure $2(\mathrm{a})$ ), $\mathrm{NAD}^{+}$might be produced by the inner membrane $\mathrm{H}^{+}$transhydrogenase from NADH with the simultaneous formation of NADPH from $\mathrm{NADP}^{+}$in the matrix [67], thereby activating SIRT3mediated IDH2 deacetylation. Moreover, the usual acetylation of proteins may be retarded in highly glycolytic cancer cells; hence, no SIRT3-mediated deacetylation would be required.

3.3. Intermittent Nature of RCG. Unlike glycolysis, RCG does not form ATP. Hence, either RCG coexistence with glycolysis or intermittent glycolysis is expected under hypoxic and deep hypoxic conditions [1]; that is, RCG may help cancer cells survive aglycemia and hypoxia in malignant cells [68]. Nevertheless, before all ATP stores become consumed, glycolysis must be reestablished. If this happens, it may help the tumor cell survive even in anoxia. As clearly demonstrated by the examples of gliomas and AML with the oncogenic metabolite D-2-hydroxyglutarate, the establishment of RCG, even concomitantly with OXPHOS glutaminolysis (note that this mode does not require IDH2 and aconitase reactions), helps to accelerate the malignant phenotype. Recently, it has been demonstrated that hypoxia elevates RCG in SF188 cells in a HIF-dependent manner [41]. SF188 cells were able to proliferate at $0.5 \% \mathrm{O}_{2}$ even if such hypoxic conditions substantially diminished glucose-dependent production of citrate, that is, OXPHOS and forward Krebs cycle participation [41].

\section{Role of IDH2 in ROS Homeostasis}

4.1. Regulation of ROS Homeostasis in Nonmalignant Cells. A major function of IDH2 in nonmalignant cells, when acting within the forward Krebs cycle, is likely maintaining an adequate pool of reduced glutathione and peroxiredoxin by providing NADPH. This function improves the mitochondrial redox balance and prevents oxidative damage [45, 46,69 ], including heat-shock-induced oxidative damage [70] and numerous consequent events of oxidative stress, such as 
ROS-induced apoptosis [71, 72], apoptosis induced by ionizing radiation [73] and cadmium [74], and staurosporineinduced cell death [75]. The lack of IDH2 or its activity elevates cytosolic ROS, lipid peroxidation, and oxidative DNA damage and shortens cell survival after oxidant exposure [69, 71-73]. Also, susceptibility to curcumin-induced apoptosis has been demonstrated upon IDH2 silencing in HCT116 cells [76]. Cardiac hypertrophy development is attributed to a decrease in IDH2 activity owing to the lipoperoxidation product 4-hydroxynonenal and oxidative stress [77]. IDH2 is also protective for paraquat-mediated oxidative inactivation of aconitase in heart mitochondria [78]. Inactivation of IDH2 activity by various ROS insults [79] is an important factor that has to be accounted for in any consideration of oxidative stress in cells. The forward Krebs cycle activity of IDH2 is inactivated by 4-hydroxynonenal [80], singlet oxygen [81], hypochlorous acid [82], aluminum [83], nitric oxide [84], and peroxynitrite [85]. Peroxynitrite forms Snitrosothiol adducts on Cys305 and Cys387 of IDH2 under nitrosative stress, such as that established in the liver of ethanol-fed rats [85]. Glycation-mediated IDH2 damage has also been reported [86].

IDH2 activity first increases and then decreases with age in fibroblasts and liver, kidney, and testes tissues of rats fed ad libitum but not of those fed a calorie-restricted diet [87]. Recently, caloric restriction has been proven to act via IDH2 deacetylation through SIRT3 and thus promote an antioxidant role for IDH2-produced NADPH [44]. Again, the activity within the forward Krebs cycle was considered. It is not known whether SIRT3-mediated deacetylation also activates the NADPH-dependent "reverse" reaction, that is, reductive carboxylation.

4.2. The Dissipative Isocitrate/2OG Cycle. The dissipative isocitrate/2OG cycle has been suggested based on the reductive carboxylation reaction of IDH2 (counter Krebs cycle reaction direction, NADPH dependent) in conjunction with the forward IDH3 reaction in the canonical Krebs cycle [63]. The cycle may manifests itself in the absence of citrate export from mitochondria, as normally occurs in non-malignant cells, since cycling is impossible when reversed aconitase reaction depletes isocitrate. The cycle would also be possible at sufficient reactant pools and inner membrane energization. Isocitrate formed by the reductive carboxylation reaction of IDH2 is processed back to $2 \mathrm{OG}$ by IDH3. Although in non-malignant cells Complex I regenerates NADH to $\mathrm{NAD}^{+}$and $\mathrm{NADP}^{+}$could be regenerated to NADPH by, for example, mitochondrial malic enzyme, with increasing malignancy (more dormant state of mitochondria and hence decreasing respiration), the mitochondrial inner membrane $\mathrm{H}^{+}$transhydrogenase $[24,63,67]$ may alternatively transfer electrons from $\mathrm{NADH}$ and $\mathrm{NADP}^{+}$to $\mathrm{NAD}^{+}$and NADPH at the expense of the proton-motive force [24]. However, it remains to be determined, whether this cycle is possible with $\mathrm{D}$-2-hydroxyglutarate. If $\mathrm{D}$-2hydroxyglutarate was metabolized by IDH3 in the canonical Krebs cycle, then the cycle would be automatically induced by the appearance of D-2-hydroxyglutarate at simultaneously active $\mathrm{H}^{+}$transhydrogenase. Nevertheless OXPHOS cannot be completely dormant, since proton-motive force would be required for this normal "forward" transhydrogenase reaction [24].

\subsection{Consequences of Reductive Carboxylation for ROS Home-} ostasis in Cancer Cells. Consider the situation in highly malignant cells in which energy is derived primarily from glycolysis disconnected from OXPHOS (Warburg phenotype) and high reductive carboxylation glutaminolysis takes place (Figure 2(a)). Presumably, OXPHOS impairment [2] or deep hypoxia [41] may set up this metabolic pattern. In this case, higher glucose-6-phosphate dehydrogenase activity (the first PPP enzyme) produces more NADPH [88]. It may be erroneously considered as antioxidant action; however, because the constitutively expressed NADPH oxidase isoform-4, NOX4 $[89,90]$, can consume a major portion of the excess NADPH and produce more superoxide and consequently release more $\mathrm{H}_{2} \mathrm{O}_{2}$ into the cytosol, the overall reaction scheme may be prooxidant (Figure 2(a)). This contributes to a much higher oxidative stress state in highly malignant cancer cells. Recently, NOX4 was also suggested to have mitochondrial localization [91]. Probably, NOX4 has $\mathrm{K}_{m}$ in the same order of magnitude as IDH enzymes. The NOX4 consumption of NADPH leaves fewer redox equivalents for the reduction of cellular glutathione and other redox systems [92-94]. The cytosolic oxidative stress is further intensified by the slow electron transport in low respiring (dormant) mitochondria of highly malignant cancer cells [1$5,68]$, resulting in more superoxide release to the cytosol as well as the mitochondrial matrix from the respiratory chain. The ongoing maximum reductive carboxylation reaction further contributes to the oxidative stress by consuming $\mathrm{NADPH}$, thus leaving less NADPH for maintenance of the reduced glutathione pool. Moreover, as mentioned above, the accumulated NADH at slow respiration may lead to $\mathrm{NAD}^{+}$formation in the reversed $\mathrm{H}^{+}$transhydrogenase reaction by concomitant NADPH formation from $\mathrm{NADP}^{+}$ to further feed the NADPH pool and hence reductive carboxylation. Simultaneously, $\mathrm{NAD}^{+}$may lead to SIRT3mediated activation of IDH2, at least of its "forward mode" [44], but it is not known whether reductive carboxylation is also activated by deacetylation of IDH2.

4.4. Consequences of IDH2 Reaction within the Forward Krebs Cycle for ROS Homeostasis in Cancer Cells. We next consider an intermediate Warburg phenotype, characterized by the mixed use of sole glycolysis, that is, aerobic glycolysis producing lactate, and OXPHOS (Figure 2(b)). The latter may be represented either by OXPHOS pyruvate metabolism and/or by OXPHOS glutaminolysis. Under these conditions, considerable cytosolic oxidative stress is expected because of the elevated NOX4 activity, as described above. However, a lower mitochondrial contribution to the cytosolic oxidative stress exists owing to an intermediate level of respiration and hence lower superoxide release from mitochondria to both the cytosolic and matrix compartments [92]. There is also lower oxidative stress expected in the matrix owing to the 


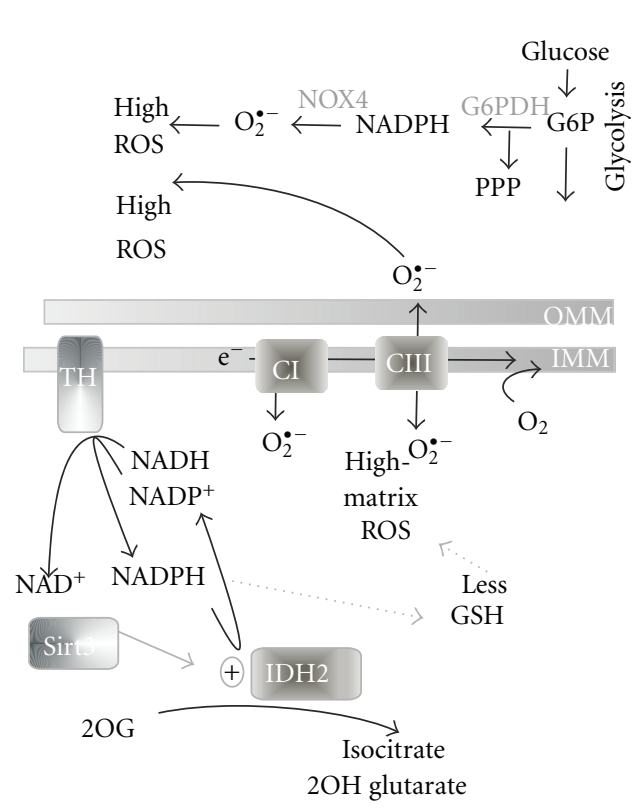

(a)

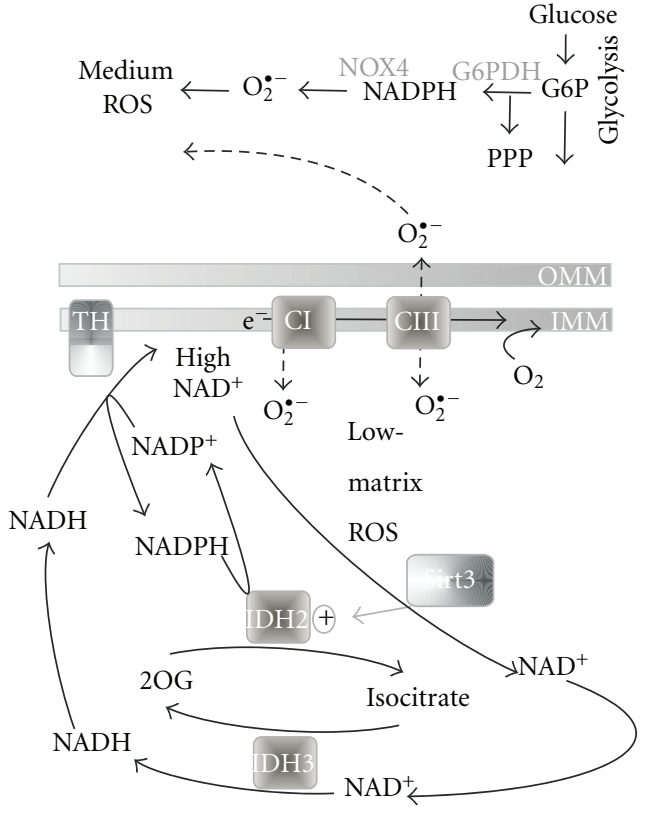

(b)

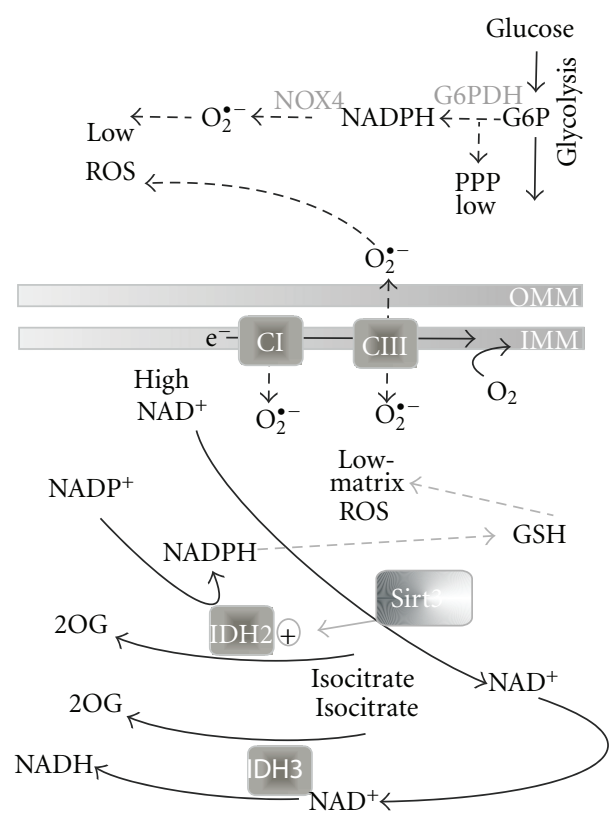

(c)

FIGURE 2: Consequences of IDH2 functions for redox homeostasis in cancer cells and nonmalignant cells.

possible ongoing dissipative isocitrate/2OG cycle, which by decreasing the proton-motive force [63] decreases mitochondrial superoxide formation [95]. This can be considered, however, only when citrate efflux from mitochondria is not dominant or when D-2-hydroxyglutarate would be cycling instead of isocitrate/2OG. If this is the case, $\mathrm{NAD}^{+}$rather than $\mathrm{NADH}$ would accumulate, further feeding the isocitrate/2OG cycle by simultaneous action of the forward IDH3 reaction and reverse (reductive carboxylation) reaction of IDH2. The accumulated $\mathrm{NAD}^{+}$would also promote SIRT3mediated IDH2 deacetylation, consequently accelerating the
IDH2 branch of the reaction cycle (Figure 2(b)). Conditions similar to those described here may occur at hypoxia.

4.5. Regulation of ROS Homeostasis in Nonmalignant Cells: IDH2 Contribution. Finally, we consider the situation in non-malignant cells, in which OXPHOS predominates and the sole glycolysis and PPP activities are low (Figure 2(c)). In this case, low oxidative stress in the cytosol is a consequence of the negligible NOX4 activity and the low contribution of mitochondria to the cytosolic ROS pool. Under these normal 
conditions, we assume that the forward IDH2 reaction generates NADPH, which further improves the reduced state of the mitochondrial matrix glutathione and peroxiredoxin systems. Indeed, ample evidence suggests that in cells with unattenuated OXPHOS, IDH2 plays an important antioxidant role that is further strengthened by $\mathrm{NAD}^{+}$accumulation in highly respiring cells. $\mathrm{NAD}^{+}$then induces SIRT3mediated IDH2 deacetylation, thus increasing its protective function in NADPH formation for the maintenance of the reduced glutathione and peroxiredoxin systems and for selfmaintenance by the reactivation of cystine-inactivated IDH2 by glutaredoxin-2 [59].

4.6. IDH2-Dependent ROS Homeostasis at Glance. (a) Situation in cancer cells with a prevalent Warburg phenotype and high reductive carboxylation. In the cytosol, higher glucose6-phosphate dehydrogenase activity (G6PDH) produces higher NADPH within the pentose phosphate pathway (PPP). NADPH-oxidase isoform-4 (NOX4) thus produces more superoxide and consequently contributes to high levels of reactive oxygen species (ROS) in the cytosol. The cytosolic oxidative stress is further intensified by the slow electron transport in low-respiring (dormant) mitochondria, leading to higher superoxide release to the cytosol and matrix compartments. The ongoing maximum reductive carboxylation reaction further contributes to oxidative stress by consuming $\mathrm{NADPH}$, thus leaving less for maintenance of the reduced glutathione pool. The accumulated $\mathrm{NADH}$ at slow respiration may lead to $\mathrm{NAD}^{+}$formation in the reverse $\mathrm{H}^{+}$ transhydrogenase $(\mathrm{TH})$ reaction (due to low proton/motive force) with concomitant NADPH formation from $\mathrm{NADP}^{+}$ to further feed the NADPH pool and hence reductive carboxylation. Hypothetically, $\mathrm{NAD}^{+}$may lead to sirtuin 3 (SIRT3)-mediated deacetylation/activation (+)of IDH2, but it is not known whether reductive carboxylation is also activated by deacetylation of IDH2. (b) Situation in cancer cells with an intermediate Warburg phenotype and possible OXPHOS glutaminolysis. The major contribution to the cytosolic oxidative stress is the same as described above. However, a lower mitochondrial contribution to cytosolic ROS leads to intermediate oxidative stress under these conditions. Indeed, an intermediate or high respiration leads to much lower superoxide production and release from the mitochondria to both the cytosolic and matrix compartments (dashed arrows). Lower oxidative stress is also expected in the matrix owing to the ongoing dissipative isocitrate/2OG cycle, which decreases further mitochondrial superoxide formation by decreasing the proton-motive force. OXPHOS glutaminolysis may predominate under these conditions; hence, RCG might not be completed and the isocitrate/2OG cycle with forward $\mathrm{H}^{+}$transhydrogenase reaction may be initiated. $\mathrm{NAD}^{+}$would accumulate owing to high respiration, further feeding the isocitrate/2OG cycle. The accumulated $\mathrm{NAD}^{+}$would also promote SIRT3mediated deacetylation/activation of IDH2. (c) Situation in nonmalignant cells. Aerobic glycolysis and PPP activity are low; consequently, low oxidative stress in the cytosol also results from the negligible NOX4 activity and the low contribution of mitochondria to the cytosolic ROS. The forward IDH2 reaction thus forms NADPH, which further improves the reduced state in the mitochondrial matrix glutathione and peroxiredoxin systems. As in (b), the NAD ${ }^{+}$ accumulation in highly respiring cells then induces SIRT3mediated deacetylation/activation of IDH2.

\section{Hypothetical IDH2 Involvement in Redox Signaling}

As briefly described above, mutant IDH2 as well as IDH1 [96-100] produce D-2-hydroxy-glutarate, which can initiate an HIF-mediated "hypoxic type" of gene reprogramming even at normoxia [58]. Nevertheless, detail investigations of D-2-hydroxyglutarate effects on HIF signaling are required, since recently an opposite effect, diminishing HIF levels by inhibition of EGLN prolyl 4-hydroxylases, has been reported [99]. Also, non-mutated IDH2 acting in the 2OG/isocitrate cycle together with $\mathrm{H}^{+}$transhydrogenase [63] could contribute to the modulation of the ROS pool by initiating an impulse originating from Complex III to dissipate the proton-motive force, which reduces superoxide formation in the mitochondrial respiratory chain [95]. In contrast to mutant IDH2 activity, the ongoing dissipative 2OG/isocitrate cycle in the absence of citrate efflux from mitochondria would retard HIF signaling.

\section{Future Perspectives}

The discovery of mutant IDH2 and IDH1 in certain gliomas and AML and their production of the oncogenic metabolite D-2-hydroxyglutarate have unraveled a fascinating story of cancer self-acceleration via intermittent episodes of genome instability and metabolic remodeling and namely via epigenomic alterations $[98,100]$. However, there are additional aspects to be clarified. First, the exact role of D-2-hydroxyglutarate must be further investigated to determine whether it promotes the reverse carboxylation mode of glutaminolysis and whether it acts in the dissipative 2OG/isocitrate cycle, which would then become the 2OG/D-2-hydroxyglutarate cycle, as we can now only speculate. Also, conditions under which D-2-hydroxyglutarate might be formed in non-mutant IDH2 should be defined. Second, the role of SIRT3 has to be established to determine whether it prevents or accelerates malignancy via IDH2. In particular, the question whether SIRT3 activates reductive carboxylation must be resolved. Finally, the role of IDH2 in other cancer types distinct from AML, gliomas, and renal tumors of hereditary leiomyomatosis should be investigated.

\section{Acknowledgment}

This work has been supported by the Grant Agency of the Czech Republic, Grant no. P301/12/P381 to K. Smolková, and P302/10/0346 to P. Ježek. 


\section{References}

[1] K. Smolková, L. Plecitá-Hlavatá, N. Bellance, G. Benard, R. Rossignol, and P. Ježek, "Waves of gene regulation suppress and then restore oxidative phosphorylation in cancer cells," International Journal of Biochemistry and Cell Biology, vol. 43, no. 7, pp. 950-968, 2011.

[2] A. R. Mullen, W. W. Wheaton, E. S. Jin et al., "Reductive carboxylation supports growth in tumour cells with defective mitochondria," Nature, vol. 481, no. 7381, pp. 385-388, 2011.

[3] M. Yuneva, "Finding an "Achilles' heel" of cancer: the role of glucose and glutamine metabolism in the survival of transformed cells," Cell Cycle, vol. 7, no. 14, pp. 2083-2089, 2008.

[4] R. J. DeBerardinis and T. Cheng, "Q's next: the diverse functions of glutamine in metabolism, cell biology and cancer," Oncogene, vol. 29, no. 3, pp. 313-324, 2010.

[5] B. Nadege, L. Patrick, and R. Rodrigue, "Mitochondria: from bioenergetics to the metabolic regulation of carcinogenesis," Frontiers in Bioscience, vol. 14, no. 11, pp. 4015-4034, 2009.

[6] N. C. Denko, "Hypoxia, HIF1 and glucose metabolism in the solid tumour," Nature Reviews Cancer, vol. 8, no. 9, pp. 705713, 2008.

[7] P. Ježek, L. Plecitá-Hlavatá, K. Smolková, and R. Rossignol, "Distinctions and similarities of cell bioenergetics and the role of mitochondria in hypoxia, cancer, and embryonic development," International Journal of Biochemistry and Cell Biology, vol. 42, no. 5, pp. 604-622, 2010.

[8] R. J. Shaw, "Glucose metabolism and cancer," Current Opinion in Cell Biology, vol. 18, no. 6, pp. 598-608, 2006.

[9] R. J. DeBerardinis, J. J. Lum, G. Hatzivassiliou, and C. B. Thompson, "The biology of cancer: metabolic reprogramming fuels cell growth and proliferation," Cell Metabolism, vol. 7, no. 1, pp. 11-20, 2008.

[10] M. Yuneva, N. Zamboni, P. Oefner, R. Sachidanandam, and Y. Lazebnik, "Deficiency in glutamine but not glucose induces MYC-dependent apoptosis in human cells," Journal of Cell Biology, vol. 178, no. 1, pp. 93-105, 2007.

[11] T. Cheng, J. Sudderth, C. Yang et al., "Pyruvate carboxylase is required for glutamine-independent growth of tumor cells," Proceedings of the National Academy of Sciences of the United States of America, vol. 108, no. 21, pp. 8674-8679, 2011.

[12] P. Gao, I. Tchernyshyov, T. C. Chang et al., "C-Myc suppression of miR-23a/b enhances mitochondrial glutaminase expression and glutamine metabolism," Nature, vol. 458, no. 7239, pp. 762-765, 2009.

[13] D. R. Wise, R. J. DeBerardinis, A. Mancuso et al., "Myc regulates a transcriptional program that stimulates mitochondrial glutaminolysis and leads to glutamine addiction," Proceedings of the National Academy of Sciences of the United States of America, vol. 105, no. 48, pp. 18782-18787, 2008.

[14] R. J. DeBerardinis, A. Mancuso, E. Daikhin et al., "Beyond aerobic glycolysis: transformed cells can engage in glutamine metabolism that exceeds the requirement for protein and nucleotide synthesis," Proceedings of the National Academy of Sciences of the United States of America, vol. 104, no. 49, pp. 19345-19350, 2007.

[15] R. W. Moreadith and A. L. Lehninger, "The pathways of glutamate and glutamine oxidation by tumor cell mitochondria. Role of mitochondrial NAD $(\mathrm{P})+$-dependent malic enzyme," The Journal of Biological Chemistry, vol. 259, no. 10, pp. 6215-6221, 1984.

[16] R. W. Moreadith and A. L. Lehninger, "Purification, kinetic behavior, and regulation of $\mathrm{NAD}(\mathrm{P})+$ malic enzyme of tumor mitochondria," The Journal of Biological Chemistry, vol. 259, no. 10, pp. 6222-6227, 1984.

[17] M. Israël and L. Schwartz, "The metabolic advantage of tumor cells," Molecular Cancer, vol. 10, article 70, 2011.

[18] R. Flavin, S. Peluso, P. L. Nguyen, and M. Loda, "Fatty acid synthase as a potential therapeutic target in cancer," Future Oncology, vol. 6, no. 4, pp. 551-562, 2010.

[19] J. A. Menendez, "Fine-tuning the lipogenic/lipolytic balance to optimize the metabolic requirements of cancer cell growth: molecular mechanisms and therapeutic perspectives," Biochimica et Biophysica Acta, vol. 1801, no. 3, pp. 381391, 2010.

[20] L. J. Reitzer, B. M. Wice, and D. Kennell, "Evidence that glutamine, not sugar, is the major energy source for cultured HeLa cells," The Journal of Biological Chemistry, vol. 254, no. 8, pp. 2669-2676, 1979.

[21] A. L. Holleran, D. A. Briscoe, G. Fiskum, and J. K. Kelleher, "Glutamine metabolism in AS-30D hepatoma cells. Evidence for its conversion into lipids via reductive carboxylation," Molecular and Cellular Biochemistry, vol. 152, no. 2, pp. 95101, 1995.

[22] H. Yoo, M. R. Antoniewicz, G. Stephanopoulos, and J. K. Kelleher, "Quantifying reductive carboxylation flux of glutamine to lipid in a brown adipocyte cell line," The Journal of Biological Chemistry, vol. 283, no. 30, pp. 20621-20627, 2008.

[23] P. S. Ward, J. Patel, D. R. Wise et al., "The common feature of leukemia-associated $I D H 1$ and $I D H 2$ mutations is a neomorphic enzyme activity converting alpha-ketoglutarate to 2-hydroxyglutarate," Cancer Cell, vol. 17, no. 3, pp. 225-234, 2010.

[24] S. P. Albracht, A. J. Meijer, and J. Rydström, "Mammalian NADH:ubiquinone oxidoreductase (Complex I) and nicotinamide nucleotide transhydrogenase $(\mathrm{Nnt})$ together regulate the mitochondrial production of $\mathrm{H}_{2} \mathrm{O}_{2}$-implications for their role in disease, especially cancer," Journal of Bioenergetics and Biomembranes, vol. 43, no. 5, pp. 541-564, 2011.

[25] R. M. Denton, "Regulation of mitochondrial dehydrogenases by calcium ions," Biochimica et Biophysica Acta, vol. 1787, no. 11, pp. 1309-1316, 2009.

[26] Z. J. Reitman and H. Yan, "Isocitrate dehydrogenase 1 and 2 mutations in cancer: alterations at a crossroads of cellular metabolism," Journal of the National Cancer Institute, vol. 102, no. 13, pp. 932-941, 2010.

[27] L. Dang, S. Jin, and S. M. Su, "IDH mutations in glioma and acute myeloid leukemia," Trends in Molecular Medicine, vol. 16, no. 9, pp. 392-397, 2010.

[28] M. F. Amary, K. Bacsi, F. Maggiani et al., "IDH1 and IDH2 mutations are frequent events in central chondrosarcoma and central and periosteal chondromas but not in other mesenchymal tumours," Journal of Pathology, vol. 224, no. 3, pp. 334-343, 2011.

[29] D. Capper, M. Simon, C. D. Langhans et al., "2-Hydroxyglutarate concentration in serum from patients with gliomas does not correlate with IDH1/2 mutation status or tumor size," International Journal of Cancer. In press.

[30] F. Ducray, Y. Marie, and M. Sanson, "IDH1 and IDH2 mutations in gliomas," The New England Journal of Medicine, vol. 360, no. 21, pp. 2248-2249, 2009.

[31] C. Guo, C. J. Pirozzi, G. Y. Lopez, and H. Yan, "Isocitrate dehydrogenase mutations in gliomas: mechanisms, biomarkers and therapeutic target," Current Opinion in Neurology, vol. 24 , no. 6, pp. 648-652, 2011. 
[32] C. Horbinski, L. Kelly, Y. E. Nikiforov, M. B. Durso, and M. N. Nikiforova, "Detection of IDH1 and IDH2 mutations by fluorescence melting curve analysis as a diagnostic tool for brain biopsies," Journal of Molecular Diagnostics, vol. 12, no. 4, pp. 487-492, 2010.

[33] D. Krell, M. Assoku, M. Galloway, P. Mulholland, I. Tomlinson, and C. Bardella, "Screen for IDH1, IDH2, IDH3, D2HGDH and $2 \mathrm{HGDH}$ mutations in glioblastoma," PLoS One, vol. 6, no. 5, Article ID e19868, 2011.

[34] M. Mellai, A. Piazzi, V. Caldera et al., "IDH1 and IDH2 mutations, immunohistochemistry and associations in a series of brain tumors," Journal of Neurooncology, vol. 105, no. 2, pp. 345-357, 2011.

[35] Z. J. Reitman, G. Jin, E. D. Karoly et al., "Profiling the effects of isocitrate dehydrogenase 1 and 2 mutations on the cellular metabolome," Proceedings of the National Academy of Sciences of the United States of America, vol. 108, no. 8, pp. 3270-3275, 2011.

[36] P. S. Ward, J. R. Cross, C. Lu et al., "Identification of additional IDH mutations associated with oncometabolite R(-)-2-hydroxyglutarate production," Oncogene. In press.

[37] H. Yan, D. W. Parsons, G. Jin et al., "IDH1 and IDH2 mutations in gliomas," The New England Journal of Medicine, vol. 360, no. 8, pp. 765-773, 2009.

[38] S. Gross, R. A. Cairns, M. D. Minden et al., "Cancer-associated metabolite 2-hydroxyglutarate accumulates in acute myelogenous leukemia with isocitrate dehydrogenase 1 and 2 mutations," Journal of Experimental Medicine, vol. 207, no. 2, pp. 339-344, 2010.

[39] G. Jin, Z. J. Reitman, I. Spasojevic et al., "2-hydroxyglutarate production, but not dominant negative function, is conferred by glioma-derived $\mathrm{NADP}^{+}$-dependent isocitrate dehydrogenase mutations," PLoS One, vol. 6, no. 2, Article ID e16812, 2011.

[40] W. Xu, H. Yang, Y. Liu et al., "Oncometabolite 2-hydroxyglutarate is a competitive inhibitor of $\alpha$-ketoglutarate-dependent dioxygenases," Cancer Cell, vol. 19, no. 1, pp. 17-30, 2011.

[41] D. R. Wise, P. S. Ward, and J. E. Shay, "Hypoxia promotes isocitrate dehydrogenase-dependent carboxylation of $\alpha$ ketoglutarate to citrate to support cell growth and viability," Proceedings of the National Academy of Sciences USA, vol. 108, no. 49, pp. 19611-19616, 2011.

[42] C. V. Pereira, M. Lebiedzinsk, M. R. Wieckowski, and P. J. Oliveira, "Regulation and protection of mitochondrial physiology by sirtuins," Mitochondrion, vol. 12, no. 1, pp. 66-76, 2011.

[43] M. N. Sack, "Emerging characterization of the role of SIRT3 mediated mitochondrial protein deacetylation in the heart," American Journal of Physiology, vol. 301, no. 6, pp. H2191H2197, 2011.

[44] S. Someya, W. Yu, W. C. Hallows et al., "Sirt3 mediates reduction of oxidative damage and prevention of age-related hearing loss under Caloric Restriction," Cell, vol. 143, no. 5, pp. 802-812, 2010.

[45] S. H. Jo, M. K. Son, H. J. Koh et al., "Control of mitochondrial redox balance and cellular defense against oxidative damage by mitochondrial $\mathrm{NADP}^{+}$-dependent Isocitrate Dehydrogenase," The Journal of Biological Chemistry, vol. 276, no. 19, pp. 16168-16176, 2001.

[46] S. K. In and J. W. Park, "Regulation of mitochondrial $\mathrm{NADP}^{+}$-dependent isocitrate dehydrogenase activity by glutathionylation," The Journal of Biological Chemistry, vol. 280, no. 11, pp. 10846-10854, 2005.
[47] C. M. Haraguchi, T. Mabuchi, and S. Yokota, "Localization of a mitochondrial type of NADP-dependent isocitrate dehydrogenase in kidney and heart of rat: an immunocytochemical and biochemical study," Journal of Histochemistry and Cytochemistry, vol. 51, no. 2, pp. 215-226, 2003.

[48] I. U. Oh, J. Inazawa, Y. O. Kim, B. J. Song, and T. L. Huh, "Assignment of the human mitochondrial $\mathrm{NADP}^{+}$-specific isocitrate dehydrogenase (IDH2) gene to $15 \mathrm{q} 26.1$ by in situ hybridization," Genomics, vol. 38, no. 1, pp. 104-106, 1996.

[49] T. Minich, S. Yokota, and R. Dringen, "Cytosolic and mitochondrial isoforms of $\mathrm{NADP}^{+}$-dependent isocitrate dehydrogenases are expressed in cultured rat neurons, astrocytes, oligodendrocytes and microglial cells," Journal of Neurochemistry, vol. 86, no. 3, pp. 605-614, 2003.

[50] C. Ceccarelli, N. B. Grodsky, N. Ariyaratne, R. F. Colman, and B. J. Bahnson, "Crystal structure of porcine mitochondrial $\mathrm{NADP}^{+}$-dependent isocitrate dehydrogenase complexed with $\mathrm{Mn} 2+$ and isocitrate: insights into the enzyme mechanism," The Journal of Biological Chemistry, vol. 277, no. 45, pp. 43454-43462, 2002.

[51] S. Soundar, B. L. Danek, and R. F. Colman, "Identification by mutagenesis of arginines in the substrate binding site of the porcine NADP-dependent isocitrate dehydrogenase," The Journal of Biological Chemistry, vol. 275, no. 8, pp. 5606$5612,2000$.

[52] R. F. Colman, "Distances among coenzyme and metal Sites of $\mathrm{NADP}^{+}$-dependent isocitrate dehydrogenase using resonance energy transfer," Biochemistry, vol. 26, no. 15, pp. 4893-4900, 1987.

[53] Y. C. Huang and R. F. Colman, "Location of the coenzyme binding site in the porcine mitochondrial NADP-dependent isocitrate dehydrogenase," The Journal of Biological Chemistry, vol. 280, no. 34, pp. 30349-30353, 2005.

[54] P. Lee and R. F. Colman, “Thr373, Asp375, and Lys260 are in the coenzyme site of porcine NADP-dependent isocitrate dehydrogenase," Archives of Biochemistry and Biophysics, vol. 450, no. 2, pp. 183-190, 2006.

[55] S. Soundar, M. O’hagan, K. S. Fomulu, R. F. Colman, A. M. Tokheim, and B. L. Martin, "Association of calcineurin with mitochondrial proteins," Proteins, vol. 64, no. 1, pp. 28-33, 2006.

[56] X. Xu, J. Zhao, Z. Xu et al., "Structures of human cytosolic NADP-dependent isocitrate dehydrogenase reveal a novel self-regulatory mechanism of activity," The Journal of Biological Chemistry, vol. 279, no. 32, pp. 33946-33957, 2004.

[57] A. Green and P. Beer, "Somatic mutations of IDH1 and IDH2 in the leukemic transformation of myeloproliferative neoplasms," The New England Journal of Medicine, vol. 362, no. 4, pp. 369-370, 2010.

[58] S. Zhao, Y. Lin, W. Xu et al., "Glioma-derived mutations in $I D H 1$ dominantly inhibit $I D H 1$ catalytic activity and induce HIF-1 $\alpha$," Science, vol. 324, no. 5924, pp. 261-265, 2009.

[59] C. A. Chrestensen, D. W. Starke, and J. J. Mieyal, "Acute cadmium exposure inactivates thioltransferase (Glutaredoxin), inhibits intracellular reduction of protein-glutathionyl-mixed disulfides, and initiates apoptosis," The Journal of Biological Chemistry, vol. 275, no. 34, pp. 26556-26565, 2000.

[60] S. C. Kim, R. Sprung, Y. Chen et al., "Substrate and functional diversity of lysine acetylation revealed by a proteomics survey," Molecular Cell, vol. 23, no. 4, pp. 607-618, 2006.

[61] S. Zhao, W. Xu, W. Jiang et al., "Regulation of cellular metabolism by protein lysine acetylation," Science, vol. 327, no. 5968, pp. 1000-1004, 2010. 
[62] C. Schlicker, M. Gertz, P. Papatheodorou, B. Kachholz, C. F. W. Becker, and C. Steegborn, "Substrates and regulation mechanisms for the human mitochondrial sirtuins Sirt3 and Sirt5," Journal of Molecular Biology, vol. 382, no. 3, pp. 790801, 2008.

[63] L. A. Sazanov and J. B. Jackson, "Proton-translocating transhydrogenase and NAD- and NADP-linked isocitrate dehydrogenases operate in a substrate cycle which contributes to fine regulation of the tricarboxylic acid cycle activity in mitochondria," FEBS Letters, vol. 344, no. 2-3, pp. 109-116, 1994.

[64] C. Des Rosiers, C. A. Fernandez, F. David, and H. Brunengraber, "Reversibility of the mitochondrial isocitrate dehydrogenase reaction in the perfused rat liver. Evidence from isotopomer analysis of citric acid cycle intermediates," The Journal of Biological Chemistry, vol. 269, no. 44, pp. 2717927182, 1994.

[65] B. Comte, G. Vincent, B. Bouchard, M. Benderdour, and C. D. Rosiers, "Reverse flux through cardiac $\mathrm{NADP}^{+}$-isocitrate dehydrogenase under normoxia and ischemia," American Journal of Physiology, vol. 283, no. 4, pp. H1505-H1514, 2002.

[66] J. M. S. Lemons, H. A. Coller, X. J. Feng et al., "Quiescent fibroblasts exhibit high metabolic activity," PLoS Biology, vol. 8, no. 10, Article ID e1000514, 2010.

[67] A. Pedersen, G. B. Karlsson, and J. Rydström, "Protontranslocating transhydrogenase: an update of unsolved and controversial issues," Journal of Bioenergetics and Biomembranes, vol. 40, no. 5, pp. 463-473, 2008.

[68] K. Smolková, N. Bellance, F. Scandurra et al., "Mitochondrial bioenergetic adaptations of breast cancer cells to aglycemia and hypoxia," Journal of Bioenergetics and Biomembranes, vol. 42, no. 1, pp. 55-67, 2010.

[69] I. S. Kil, S. Y. Kim, S. J. Lee, and J. W. Park, "Small interfering RNA-mediated silencing of mitochondrial $\mathrm{NADP}^{+}$dependent isocitrate dehydrogenase enhances the sensitivity of HeLa cells toward tumor necrosis factor- $\alpha$ and anticancer drugs," Free Radical Biology and Medicine, vol. 43, no. 8, pp. 1197-1207, 2007.

[70] H. J. Kim, B. S. Kang, and J. W. Park, "Cellular defense against heat shock-induced oxidative damage by mitochondrial $\mathrm{NADP}^{+}$-dependent isocitrate dehydrogenase," Free Radical Research, vol. 39, no. 4, pp. 441-448, 2005.

[71] A. H. Shin, I. S. Kil, E. S. Yang, T. L. Huh, C. H. Yang, and J. W. Park, "Regulation of high glucose-induced apoptosis by mitochondrial $\mathrm{NADP}^{+}$-dependent isocitrate dehydrogenase," Biochemical and Biophysical Research Communications, vol. 325, no. 1, pp. 32-38, 2004.

[72] S. W. Shin, I. S. Kil, and J. W. Park, "Silencing of mitochondrial $\mathrm{NADP}^{+}$-dependent isocitrate dehydrogenase by small interfering RNA enhances heat shock-induced apoptosis," Biochemical and Biophysical Research Communications, vol. 366, no. 4, pp. 1012-1018, 2008.

[73] H. L. Jin, Y. K. Sung, S. K. In, and J. W. Park, "Regulation of ionizing radiation-induced apoptosis by mitochondrial $\mathrm{NADP}^{+}$-dependent isocitrate dehydrogenase," The Journal of Biological Chemistry, vol. 282, no. 18, pp. 13385-13394, 2007.

[74] S. K. In, W. S. Seoung, S. Y. Hyun, S. L. Young, and J. W. Park, "Mitochondrial NADP ${ }^{+}$-dependent isocitrate dehydrogenase protects cadmium-induced apoptosis," Molecular Pharmacology, vol. 70, no. 3, pp. 1053-1061, 2006.

[75] S. J. Kim, T. Y. Yune, C. T. Han et al., "Mitochondrial isocitrate dehydrogenase protects human neuroblastoma
SH-SY5Y cells against oxidative stress," Journal of Neuroscience Research, vol. 85, no. 1, pp. 139-152, 2007.

[76] K. H. Jung and J. W. Park, "Suppression of mitochondrial $\mathrm{NADP}^{+}$-dependent isocitrate dehydrogenase activity enhances curcumin-induced apoptosis in HCT116 cells," Free Radical Research, vol. 45, no. 4, pp. 431-438, 2011.

[77] M. Benderdour, G. Charron, B. Comte et al., "Decreased cardiac mitochondrial $\mathrm{NADP}^{+}$-isocitrate dehydrogenase activity and expression: a marker of oxidative stress in hypertrophy development," American Journal of Physiology, vol. 287, no. 5, pp. H2122-H2131, 2004.

[78] K. Murakami, M. Haneda, T. Makino, and M. Yoshino, "Protective effect of NADP-isocitrate dehydrogenase on the paraquat-mediated oxidative inactivation of aconitase in heart mitochondria," Environmental Toxicology and Pharmacology, vol. 22, no. 2, pp. 148-152, 2006.

[79] S. M. Lee, T. L. Huh, and J. W. Park, "Inactivation of $\mathrm{NADP}^{+}$-dependent isocitrate dehydrogenase by reactive oxygen species," Biochimie, vol. 83, no. 11-12, pp. 1057-1065, 2001.

[80] M. Benderdour, G. Charron, D. DeBlois, B. Comte, and C. Des Rosiers, "Cardiac mitochondrial $\mathrm{NADP}^{+}$-isocitrate dehydrogenase is inactivated through 4-hydroxynonenal adduct formation: an event that precedes hypertrophy development," The Journal of Biological Chemistry, vol. 278, no. 46, pp. 45154-45159, 2003.

[81] S. Y. Kim, J. K. Tak, and J. W. Park, "Inactivation of $\mathrm{NADP}^{+}$-dependent isocitrate dehydrogenase by singlet oxygen derived from photoactivated rose bengal," Biochimie, vol. 86, no. 8, pp. 501-507, 2004.

[82] S. Young Park, S. M. Lee, S. Woo Shin, and J. W. Park, "Inactivation of mitochondrial $\mathrm{NADP}^{+}$-dependent isocitrate dehydrogenase by hypochlorous acid," Free Radical Research, vol. 42, no. 5, pp. 467-473, 2008.

[83] K. Murakami and M. Yoshino, "Aluminum decreases the glutathione regeneration by the inhibition of NADP-isocitrate dehydrogenase in mitochondria," Journal of Cellular Biochemistry, vol. 93, no. 6, pp. 1267-1271, 2004.

[84] E. S. Yang, C. Richter, J. S. Chun, T. L. Huh, S. S. Kang, and J. W. Park, "Inactivation of $\mathrm{NADP}^{+}$-dependent isocitrate dehydrogenase by nitric oxide," Free Radical Biology and Medicine, vol. 33, no. 7, pp. 927-937, 2002.

[85] J. H. Lee, E. S. Yang, and J. W. Park, "Inactivation of NADP+ dependent isocitrate dehydrogenase by peroxynitrite: implications for cytotoxicity and alcohol-induced liver injury," The Journal of Biological Chemistry, vol. 278, no. 51, pp. 5136051371, 2003.

[86] I. S. Kil, J. H. Lee, A. H. Shin, and J. W. Park, "Glycationinduced inactivation of $\mathrm{NADP}^{+}$-dependent isocitrate dehydrogenase: implications for diabetes and aging," Free Radical Biology and Medicine, vol. 37, no. 11, pp. 1765-1778, 2004.

[87] I. S. Kil, Y. S. Lee, Y. S. Bae, T. L. Huh, and J. W. Park, "Modulation of $\mathrm{NADP}^{+}$-dependent isocitrate dehydrogenase in aging," Redox Report, vol. 9, no. 5, pp. 271-277, 2004.

[88] S. A. Gupte, R. J. Levine, R. S. Gupte et al., "Glucose-6phosphate dehydrogenase-derived NADPH fuels superoxide production in the failing heart," Journal of Molecular and Cellular Cardiology, vol. 41, no. 2, pp. 340-349, 2006.

[89] C. X. Santos, L. Y. Tanaka, J. Wosniak, and F. R. Laurindo, "Mechanisms and implications of reactive oxygen species generation during the unfolded protein response: roles of endoplasmic reticulum oxidoreductases, mitochondrial electron transport, and NADPH oxidase," Antioxidants \& Redox Signaling, vol. 11, no. 10, pp. 2409-2427, 2009. 
[90] M. Yamaura, J. Mitsushita, S. Furuta et al., "NADPH oxidase 4 contributes to transformation phenotype of melanoma cells by regulating G2-M cell cycle progression," Cancer Research, vol. 69, no. 6, pp. 2647-2654, 2009.

[91] K. Block, Y. Gorin, and H. E. Abboud, "Subcellular localization of Nox4 and regulation in diabetes," Proceedings of the National Academy of Sciences of the United States of America, vol. 106, no. 34, pp. 14385-14390, 2009.

[92] P. Ježek and L. Hlavatá, "Mitochondria in homeostasis of reactive oxygen species in cell, tissues, and organism," International Journal of Biochemistry and Cell Biology, vol. 37, no. 12, pp. 2478-2503, 2005.

[93] P. Ježek and L. Plecitá-Hlavatá, "Mitochondrial reticulum network dynamics in relation to oxidative stress, redox regulation, and hypoxia," International Journal of Biochemistry and Cell Biology, vol. 41, no. 10, pp. 1790-1804, 2009.

[94] J. C. Fernández-Checa, "Redox regulation and signaling lipids in mitochondrial apoptosis," Biochemical and Biophysical Research Communications, vol. 304, no. 3, pp. 471-479, 2003.

[95] A. Dlasková, L. Hlavatá, and P. Ježek, “Oxidative stress caused by blocking of mitochondrial Complex $\mathrm{I}^{+}$pumping as a link in aging/disease vicious cycle," International Journal of Biochemistry and Cell Biology, vol. 40, no. 9, pp. 1792-1805, 2008.

[96] L. Dang, D. W. White, S. Gross et al., "Cancer-associated IDH1 mutations produce 2-hydroxyglutarate," Nature, vol. 462, no. 7274, pp. 739-744, 2009.

[97] C. M. Metallo, P. A. Gameiro, E. L. Bell et al., "Reductive glutamine metabolism by IDH1 mediates lipogenesis under hypoxia," Nature, vol. 481, no. 7381, pp. 380-384, 2011.

[98] C. Lu, P. S. Ward, G. S. Kapoor et al., "IDH mutation impairs histone demethylation and results in a block to cell differentiation," Nature, vol. 483, pp. 474-478, 2012.

[99] P. Koivunen, S. Lee, C. G. Duncan et al., "Transformation by the (R)-enantiomer of 2-hydroxyglutarate linked to EGLN activation," Nature, vol. 483, pp. 484-488, 2012.

[100] S. Turcan, D. Rohle, A. Goenka et al., "IDH1 mutation is sufficient to establish the glioma hypermethylator phenotype," Nature, vol. 483, pp. 479-483, 2012.

[101] B. Yang, C. Zhong, Y. Peng, Z. Lai, and J. Ding, "Molecular mechanisms of off-on switch of activities of human IDH1 by tumor-associated mutation R132H," Cell Research, vol. 20, no. 11, pp. 1188-1200, 2010.

[102] T. I. Rakhmanova and T. N. Popova, "Regulation of 2oxoglutarate metabolism in rat liver by NADP-isocitrate dehydrogenase and aspartate aminotransferase," Biochemistry, vol. 71, no. 2, pp. 211-217, 2006.

[103] Y. C. Huang, N. B. Grodsky, T. K. Kim, and R. F. Colman, "Ligands of the $\mathrm{Mn}^{2+}$ bound to porcine mitochondrial NADP-dependent isocitrate dehydrogenase, as assessed by mutagenesis," Biochemistry, vol. 43, no. 10, pp. 2821-2828, 2004.

[104] T. Popova, M. A. A. Pinheiro de Carvalho, L. Matasova, and L. Medvedeva, "Regulation of mitochondrial NADPisocitrate dehydrogenase in rat heart during ischemia," Molecular and Cellular Biochemistry, vol. 294, no. 1-2, pp. 97105, 2007.

[105] M. Dange and R. F. Colman, "Each conserved active site tyr in the three subunits of human isocitrate dehydrogenase has a different function," The Journal of Biological Chemistry, vol. 285, no. 27, pp. 20520-20525, 2010. 

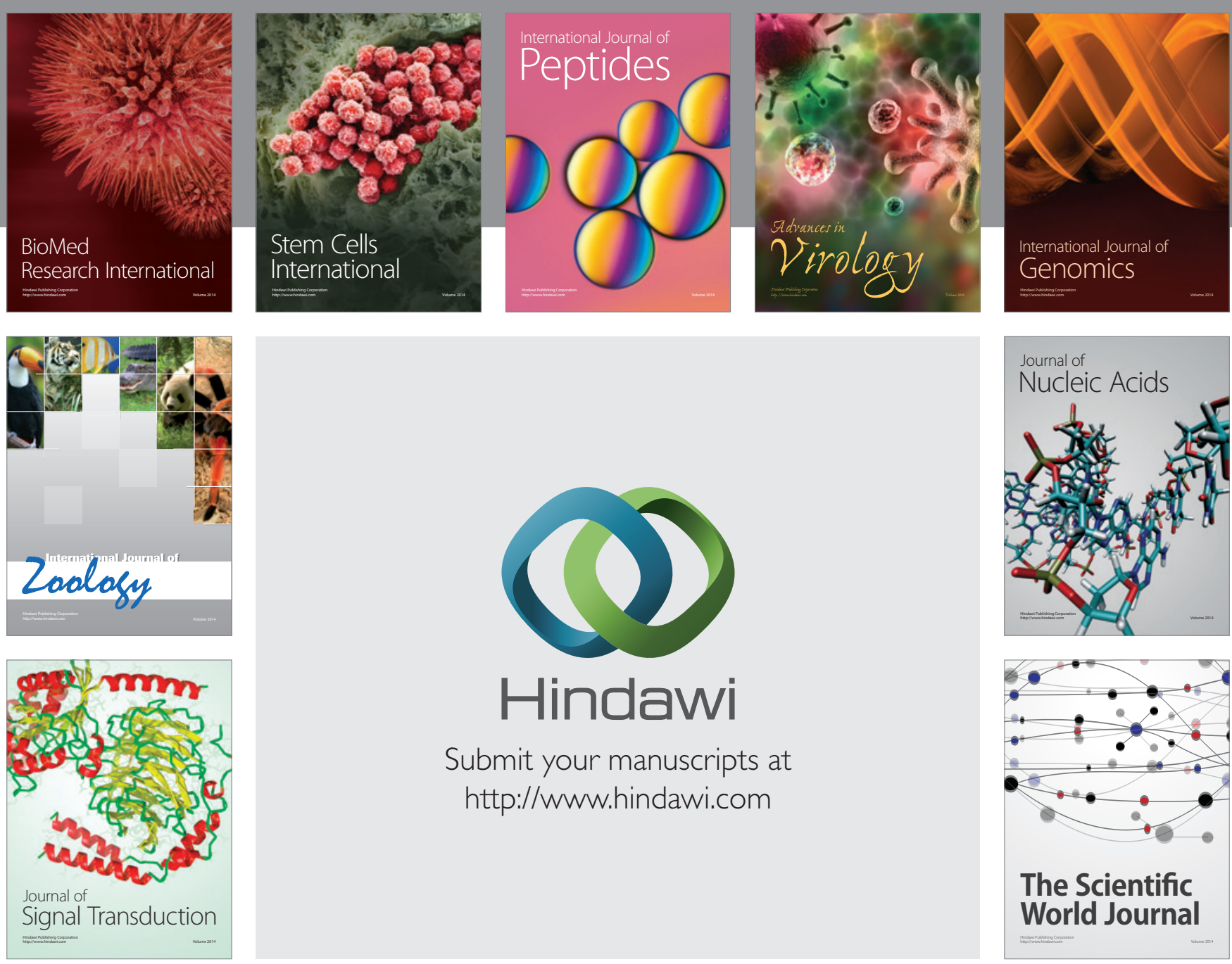

Submit your manuscripts at

http://www.hindawi.com
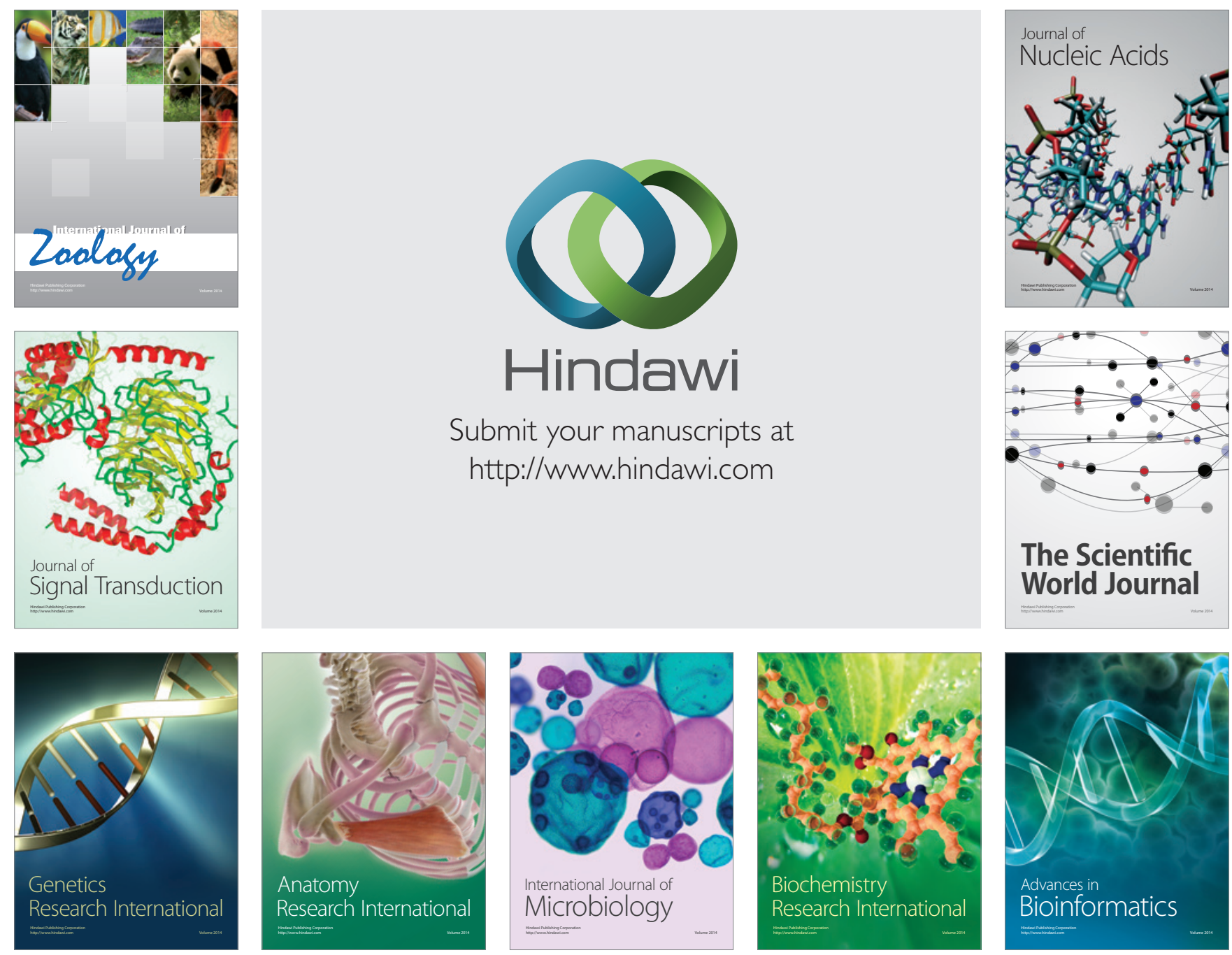

The Scientific World Journal
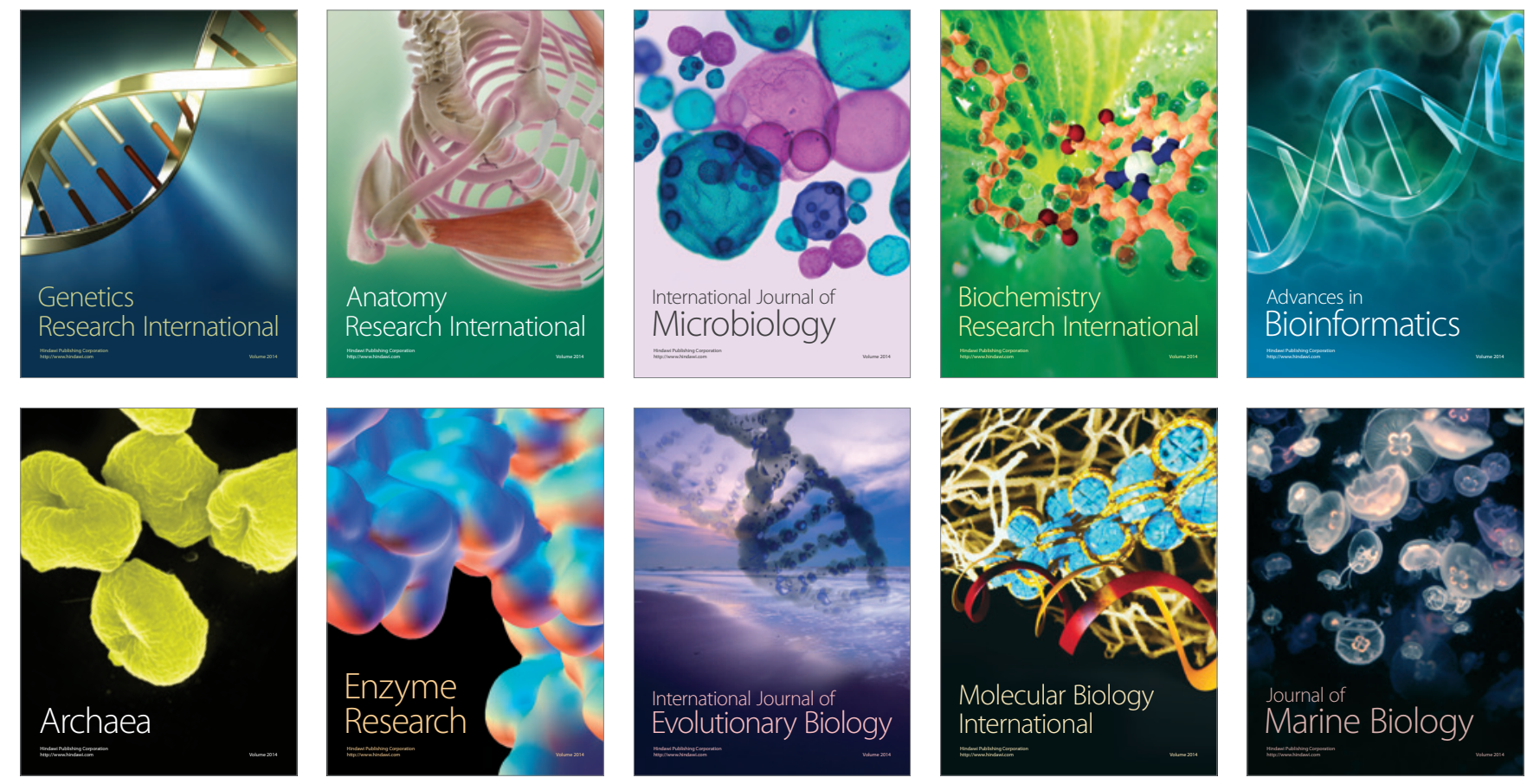